\title{
Erratum: Magnonic skin effect and magnon valve effect in an antiferromagnetically coupled heterojunction [Phys. Rev. B 104, L020413 (2021)]
}

\author{
Z. R. Yan, Y. W. Xing, and X. F. Han $\odot$
}

(Received 8 November 2021; published 24 November 2021)

DOI: 10.1103/PhysRevB.104.179901

In this erratum, we fix several typographical errors in the original paper. These simply typographical errors do not affect any results and discussions in the main text of the Letter. In the following, we list the correct expressions.

Page 1, the first term in Eq. (1) misses the permeability of vacuum $\mu_{0}$. Equation (1) should be

$$
\frac{\partial \mathbf{m}_{\eta}}{\partial t}=-\gamma \mu_{0} \mathbf{m}_{\eta} \times \mathbf{H}_{\eta}^{\text {eff }}+\alpha \mathbf{m}_{\eta} \times \frac{\partial \mathbf{m}_{\eta}}{\partial t} .
$$

Page 1, the relation in line 2 below Eq. (1) should be $\mu_{0} \mathbf{H}_{\eta}^{\text {eff }}=\sigma_{\eta} \frac{2 K_{\eta}}{M_{\eta}} \mathbf{e}_{z}+\frac{2 A_{\eta}}{M_{\eta}} \nabla^{2} \mathbf{m}_{\eta}$.

Page 2, the relation in line 2 above Eq. (2) should be $\Phi_{\eta}=m_{x, \eta}-i m_{y, \eta}$.

Page 2, the relation in line 1 below Eq. (2) should be $m_{\eta}^{*}=\hbar M_{\eta} / 4 \gamma m_{z, \eta} A_{\eta} . m_{z, \eta}$ is the $z$ component of magnetization in the $\eta$ layer. Due to $m_{z, \eta}=\sigma_{\eta}$, it can lead to $m_{\eta}^{*}=\hbar M_{\eta} / 4 \gamma m_{z, \eta} A_{\eta}=\hbar M_{\eta} / 4 \gamma \sigma_{\eta} A_{\eta}$.

Page 2, the relation in line 5 below Eq. (2) should be $E_{\eta}=\hbar \omega_{\eta}=\frac{\hbar^{2} k_{\eta}^{2}}{2 m_{\eta}^{*}}+V_{\eta}=\frac{2 \hbar \gamma \sigma_{\eta}}{M_{\eta}}\left(A_{\eta} k_{\eta}^{2}+K_{\eta}\right)$. 